\title{
Cross-Kingdom Gene Coexpression Analysis Using a Stemphylium botryosum-Lens ervoides System Revealed Plasticity of Intercommunication Between the Pathogen Secretome and the Host Immune Systems
}

\author{
Zhe Cao, and Sabine Banniza ${ }^{\dagger}$ \\ Crop Development Centre/Department of Plant Sciences, University of Saskatchewan, Saskatoon, Saskatchewan S7N 5A8, \\ Canada
}

Accepted 19 August 2021.

\begin{abstract}
Necrotrophic pathogens are responsible for significant declines in crop yield and quality worldwide. During the infection process, a pathogen releases a series of secretory proteins to counteract the plant immune system, and this interaction of pathogen and host molecules determines whether the pathogen will successfully invade the host plant tissues. In this study, we adopted co-transcriptomic approaches to analyze the Lens ervoides-Stemphylium botryosum system, with a focus on 1,216 fungal genes coding for secretory proteins and 8,810 disease-responsive genes of the host 48, 96, and $144 \mathrm{~h}$ postinoculation, captured in two $\mathrm{F}_{9}$ recombinant inbred lines (RILs) displaying contrasting disease responses. By constructing in planta gene coexpression networks (GCNs) for $S$. botryosum, we found that the pathogen tended to co-upregulate genes regulating cell wall degradation enzymes, effectors, oxidoreductases, and peptidases to a much higher degree in the susceptible host LR-66-577 than in the resistant RIL LR-66-637, indicating that the promotion of these digestive enzymes and toxins increased $S$. botryosum virulence. Construction of cross-kingdom GCNs between pathogen and plant for the two RILs revealed that the co-upregulation of these fungal digestive enzymes and toxins simultaneously promoted a series of defense responses such as redox change, expression of membrane-related genes and serine/threonine kinase, and stress and disease responses in the susceptible RIL which was not observed in the resistant RIL, indicating that these activities exacerbated susceptibility to $S$. botryosum.
\end{abstract}

Stemphylium is a genus consisting of a number of plantpathogenic species that are responsible for significant economic losses in crops worldwide. One notorious species, Stemphylium

${ }^{\dagger}$ Corresponding author: S. Banniza; sabine.banniza@usask.ca

Funding: This project was funded through an NSERC-CRD grant to S. Banniza, with industry contributions by the Saskatchewan Pulse Growers and the Western Grains Research Foundation.

*The $\boldsymbol{e}$-Xtra logo stands for "electronic extra" and indicates that a supplementary figure and supplementary tables are published online.

The author(s) declare no conflict of interest.

(c) (1) (3) () Copyright $\odot 2021$ The Author(s). This is an open access article distributed under the CC BY-NC-ND 4.0 International license. botryosum, is the causal agent of severe leaf blight in spinach (Koike et al. 2001), onion (Aveling and Snyman 1993), alfalfa (Borges et al. 1976), lentil (Mwakutuya and Banniza 2010), and clover (Graham 1957). Similar to other well-studied Stemphylium spp., S. botryosum has a classical necrotrophic lifestyle. The necrotrophic phase of this pathogen starts after its germ tubes successfully penetrate though plant epidermis cells, and secondary infectious hyphae begin to develop (Borges et al. 1976). The proliferation of $S$. botryosum significantly increases during early infection and results in an accelerated cell death visible at $48 \mathrm{~h}$ postinoculation (hpi) (Cao et al. 2019). If infection occurs under conditions favorable for the pathogen, $S$. botryosum can rapidly colonize the entire plant, causing severe leaf necrosis followed by significant defoliation. In alfalfa, Borges et al. (1976) observed that $S$. botryosum injured leaf tissue over large areas as early as 2 days postinoculation (dpi), similar to lentil, where leaf necrosis is induced by 2 dpi and severe defoliation affecting up to $80 \%$ of leaves by $7 \mathrm{dpi}$.

At the molecular level, substantial evidence has revealed that pathogens produce specialized secretory proteins to infect, colonize, acquire nutrients, and manipulate the immune system of their hosts (Guyon et al. 2014; Pandey et al. 2018). Structurally, secretory proteins are characterized by a short peptide (approximately 7 to 15 amino acids) at the $\mathrm{N}$ terminus and usually lack a hydrophobic transmembrane domain. Based on their unique structure, prediction of secretory proteins can be accomplished by several computational algorithms (Evangelisti et al. 2017). To date, a number of predictive studies have found that secretomes of necrotrophs largely consist of a diversity of plant cell wall degradation enzymes (CWDEs) targeting the cell wall polysaccharide matrix of different host species (Jing et al. 2017; Neu and Debener 2019). It has also been shown that necrotrophs can selectively use their CWDEs to degrade host wall polysaccharide substrates, depending on the type of host tissues (leave, stem, flower, and so on) (Evangelisti et al. 2017; González-Fernández et al. 2015; Morais do Amaral et al. 2012). A recent study conducted by Zeng et al. (2018) found that this is also true for S. lycopersici, because over $50 \%$ of secretory proteins were predicted to be CWDEs. In addition to CWDEs, other types of secretory proteins, including necrosis-inducing like proteins, effectors, small cysteine-rich proteins, proteases, and oxidoreductases, are also important pathogenic determinants that induce host cell necrosis, degrade defensive proteins, and distort the redox balance in hosts (Feng et al. 2014; Figaj et al. 2019; Heard et al. 2015).

In planta fungal secretory proteins of several plant-pathogen systems involving hemibiotrophs have been explored in some 
depth, revealing interesting differences in the secretomes of the distinct biotrophic and necrotrophic phases characteristic for these pathogens. In tomato, Alkan et al. (2015) found that the hemibiotroph Colletotrichum gloeosporioides greatly promoted the expression of CWDEs, including a series of celluloses and pectinases, during the necrotrophic phase. A similar expression trend was observed for a variety of oxidases and peptidases secreted by Moniliophthora roreri during its necrotrophic phase in cacao roots (Meinhardt et al. 2014). In contrast, Evangelisti et al. (2017) reported that the majority of CWDEs, effectors, and protease were downregulated during the necrotrophic stage in the Nicotiana benthamiana-Phytophthora palmivora system. Research on the necrotroph Sclerotinia sclerotiorum revealed that the regulation of some of its effectors was highly dependent on the host genotype, based on investigations of multiple Arabidopsis thaliana accessions (Guyon et al. 2014). These results together indicated that transcriptional activation and deactivation of secretory proteins are of high plasticity, depending on pathogen, host, and the interaction between these two.

Plants have evolved sophisticated innate immune systems by recruiting a variety of defensive molecules to counter the attacks from these disruptive molecules secreted by pathogens (Laluk and Mengiste 2010). When infection occurs in hosts, an intricate interplay of these attack and counterattack molecules from both parties ensues, thus creating a highly connected network of interactions, which affect the performance of both organisms (Zhang et al. 2019). The nature of these interactions will determine the outcome of the infection and the level of host resistance. Yet, knowledge of such interaction networks, especially at the genome-wide level, is scarce, primarily because of the difficulty of interconnecting the complex transcriptomes of host and pathogen.

Co-transcriptomic network analysis, which can simultaneously analyze the host and pathogen transcriptomes, provides the tools to associate actions and reactions of the two organisms together and facilitates an understanding of the interaction of two organisms. Most recently, Zhang et al. (2019) adopted this approach to study the Arabidopsis-Botrytis cinerea system and found that fungal gene expression networks involved in membrane or vesicle virulence and fungal growth were differentially linked to defense or camalexin gene networks of wild-type and Arabidopsis mutants coil-1 and nprl-1. In the same study, an association was confirmed between these cross-kingdom gene networks and the extend of lesion development on the Arabidopsis genotypes, indicating a potential use of co-transcriptomic analysis to understand the quantitative nature of disease resistance in hosts (Zhang et al. 2019).

In the present study, we employed a co-transcriptomic approach to investigate the cross-kingdom communication between Stemphylium botryosum and two Lens ervoides recombinant inbred lines (RILs) with contrasting levels of disease resistance in a temporal manner at 48, 96, and 144 hpi. Facilitated by the recently assembled $L$. culinaris genome and the availability of a range of bioinformatic tools, we had previously separated the host- pathogen transcriptome into L. ervoides- and S. botryosumderived transcriptomes in order to compare the differential transcriptomes of the two L. ervoides RILs (Cao et al. 2019). Here, we focused on the 1,216 predicted secretory genes of $S$. botryosum and their interaction with the 8,810 disease-responsive genes of both $L$. ervoides RILs, shedding light on the regulation of the $S$. botryosum secretome in response to the two host genotypes.

\section{RESULTS}

De novo assembly of the $S$. botryosum transcriptome.

We previously had mapped reads from noninoculated samples and reads from pathogen-inoculated samples (48, 96, and 192 hpi) against the $L$. culinaris genome to separate the $L$. ervoides genes from those of $S$. botryosum (Cao et al. 2019). To separate pathogen reads from host reads, a two-step filtering procedure was performed by, first, de novo assembling unmapped reads from noninoculated samples into a "leftover transcriptome" (approximately 37 million reads in size). Thereafter, reads from inoculated samples were mapped against this leftover transcriptome to eliminate the reads from $L$. ervoides, and any remaining unmapped reads were matched against the L. culinaris genome (Fig. 1A). Only the remaining unmapped and unmatched reads were recognized as enriched $S$. botryosum reads. To recover all possible in planta and time-course transcripts from this study, we then combined those enriched fungal reads from three biological replicates, three time points, and the two RILs together, which resulted in a total of 867 million reads. The de novo assembly of these recovered reads yielded a transcriptome consisting of 54,027 "Trinity genes" which corresponded to 58,040 "Trinity transcripts" (Table 1). We then assessed the completeness of the $S$. botryosum transcriptome assembly via Benchmarking Nearly Universal Single-copy Othologues (BUSCO). Among 1,315 Ascomycota BUSCOs searched, 1,181 BUSCOs (869 single-copy and 312 duplicated BUSCOs) were completely, and 101 BUSCOs were partially retrieved from our S. botryosum assembly. Only 33 BUSCOs were missing. These results indicated that our assembled $S$. botryosum transcriptome carried 97.5\% of BUSCOs genes occurring in the Ascomycota division and, hence, was considered a transcriptome of high quality and integrity that could be used as reference for the subsequent analysis.

\section{In planta gene expression of $S$. botryosum was temporally dynamic during the infection process.}

The expression of assembled genes in the form of total transcript counts and transcripts per kilobase million (TPM) were retrieved to describe the gene expression dynamics of $S$. botryosum among samples. Based on the total count of transcripts, $S$. botryosum gene expression in most samples increased along with increasing incubation time (Fig. 2A). The number of genes showing detectable expression in S. botryosum was higher in the susceptible RIL LR-66-577 than in the resistant RIL LR-66-637, especially during the late infection time points of 96 and 144 hpi. To explore the variability of $S$. botryosum gene expression among samples, gene expression in three replicate samples of the two RILs and the three sampling time points (18 samples in total) assessed as TPMs were analyzed by principal component analysis (PCA) (Fig. 2B), which separated S. botryosum samples collected at 48 hpi from others gathered at 96 and 144 hpi, confirming that gene expression of $S$. botryosum followed a clear temporal course. Plant cell viability testing showed that $S$. botryosum caused cell death at 48,96 , and $144 \mathrm{hpi}$, though the extend of necrosis varied at these time points (Fig. 2B).

\section{Secretome prediction, annotation, and gene ontology analysis.}

Transcripts of $S$. botryosum encoding secretory proteins were identified by first applying a TransDecoder-based search to predict putative open reading frames (ORFs) from a redundancy collapsed (CD-HIT) S. botryosum transcriptome (Fig. 1B). This resulted in the identification of 31,811 ORFs from assembled Trinity genes, which were analyzed with a series of tools (SignalP, TargetP, and THMHH) and a script (*KDEL or *HDEL motif removal) to predict and retain those proteins with signal peptides and localizing in secretory pathways while excluding other proteins possessing transmembrane domains or an endoplasmic reticulum (ER) signal of KDEL or HDEL at the C-terminal motif. These analyses predicted a total of 1,216 secretory proteins in the $S$. botryosum transcriptome (Supplementary Table S1). Of these, 1,020 proteins $(83.8 \%)$ were successfully annotated by at least one database (Pfam or RefSeq). 
To functionally summarize these secretory proteins, we then conducted gene ontology (GO) enrichment analysis on these proteins (Fig. 3) and identified those GO terms that were enriched in "cellular component", "biological processes", and "molecular function". The significant GO terms of "endomembrane system", "extracellular region part", "vesicle", "secretory vesicle", "extracellular exosome", and "external encapsulating structure" in the cellular component category are signatures for the involvement of secretory pathways, indicating that $S$. botryosum cells secrete proteins into the extracellular environment. The most relevant GO terms in the biological process category were carbohydrate, cell wall, lipid, and polysaccharide processes. For the molecular function category, we found that the most significant GO terms were associated with a variety of hydrolytic activities. Other GO terms of "oxidoreductase, acting on a sulfur group of donors", "cofactor transmembrane transporter activity", and "inorganic phosphate transmembrane transporter activity" in this category indicated that the pathogen had the ability to affect the host's redox status and transportation of phosphate mobility. Using twodimensional (2D)-PCA analysis, secretome expression at 96 and 144 hpi was shown to be similar; however, both could be distinguished from that at 48 hpi (Fig. 4).

\section{Hosts shaped in planta $S$. botryosum secretory gene coexpression relationship.}

Gene coexpression networks (GCNs) were constructed for fungal secretory genes to systemically investigate how $S$. botryosum regulated secretory genes in the two L. ervoides RILs possessing contrasting levels of disease resistance. For each of the two L. ervoides RILs, secretome data from the three replicates and the three time points $(48,96$, and $144 \mathrm{hpi})$ were used to calculate Spearman's rank-order correlations for all pairwise combinations of all 1,216 secretory genes. For each RIL, only highly correlated gene pairs $(R>0.9)$ were used for GCN construction. Among all GCNs, only moderate to large GCNs (size $>30$ genes) were selected for further analyses, which resulted in two GCNs for $S$. botryosum colonizing susceptible RIL LR-66577 and two for $S$. botryosum colonizing resistant RIL LR-66637 (Fig. 5; Supplementary Table S2). The overall expression trends of these GCNs were visualized in heatmaps (Fig. 6A). Results showed that the gene expression of GCN1s for both
A

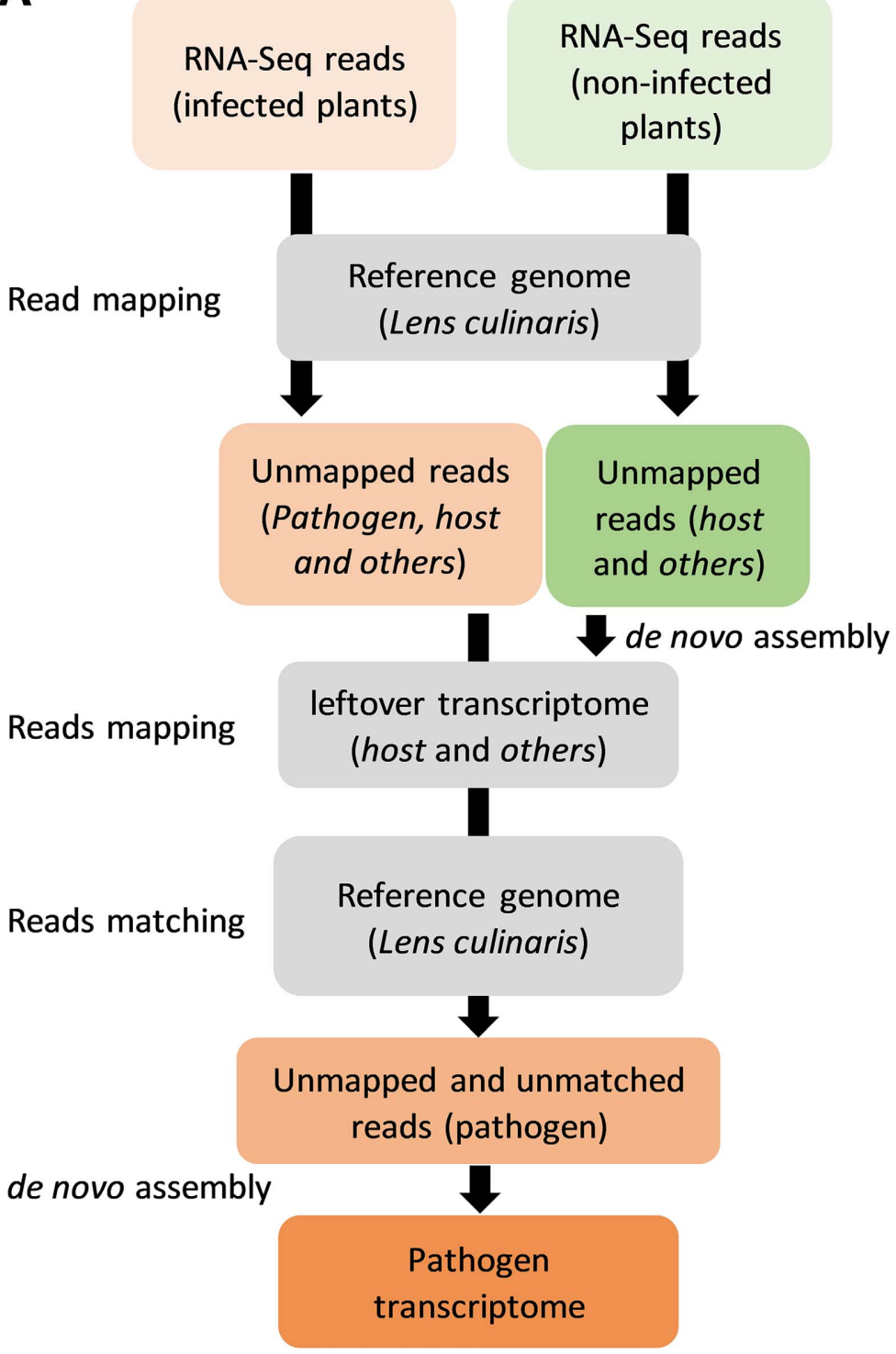

B

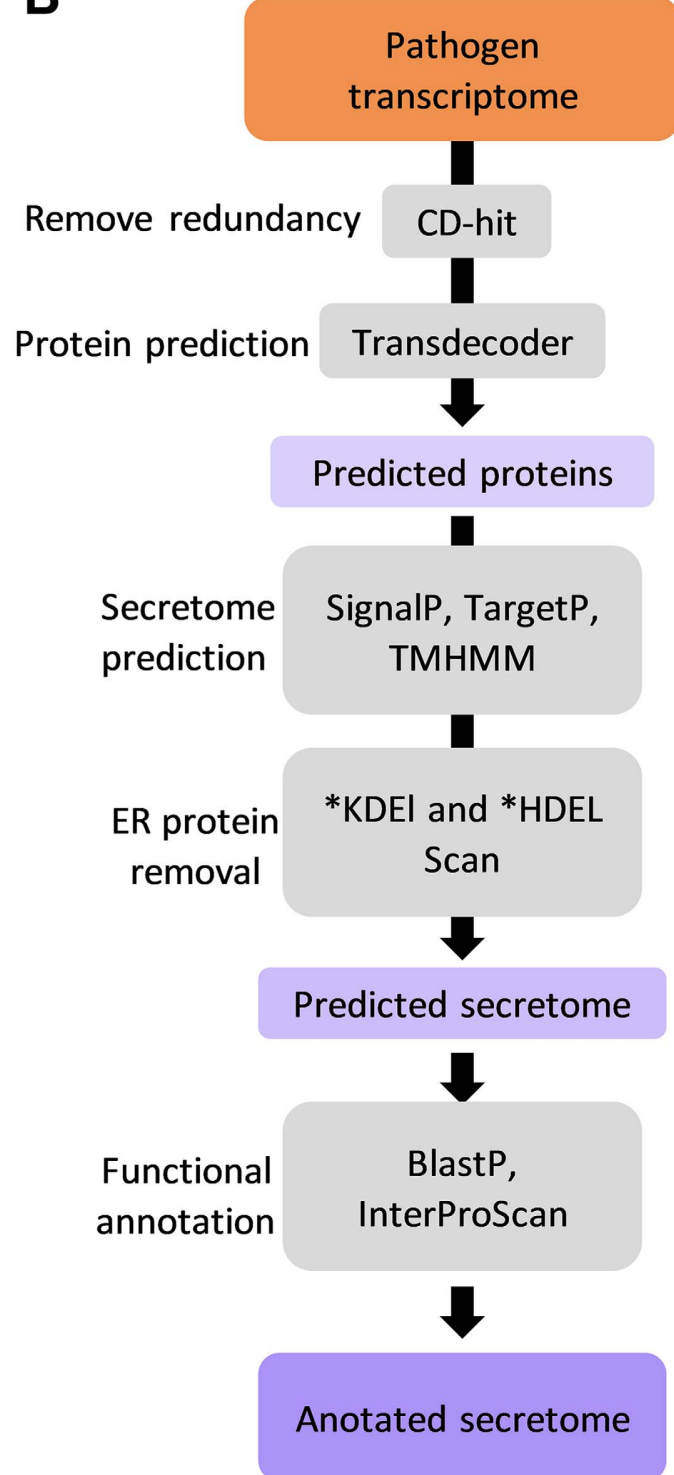

Fig. 1. Pipeline of Stemphylium botryosum sequencing data analysis. A, Separation of S. botryosum reads from the mixed transcriptome and de novo assembly of these recovered reads into an $S$. botryosum transcriptome. B, Prediction and annotation of $S$. botryosum secretome. 
A

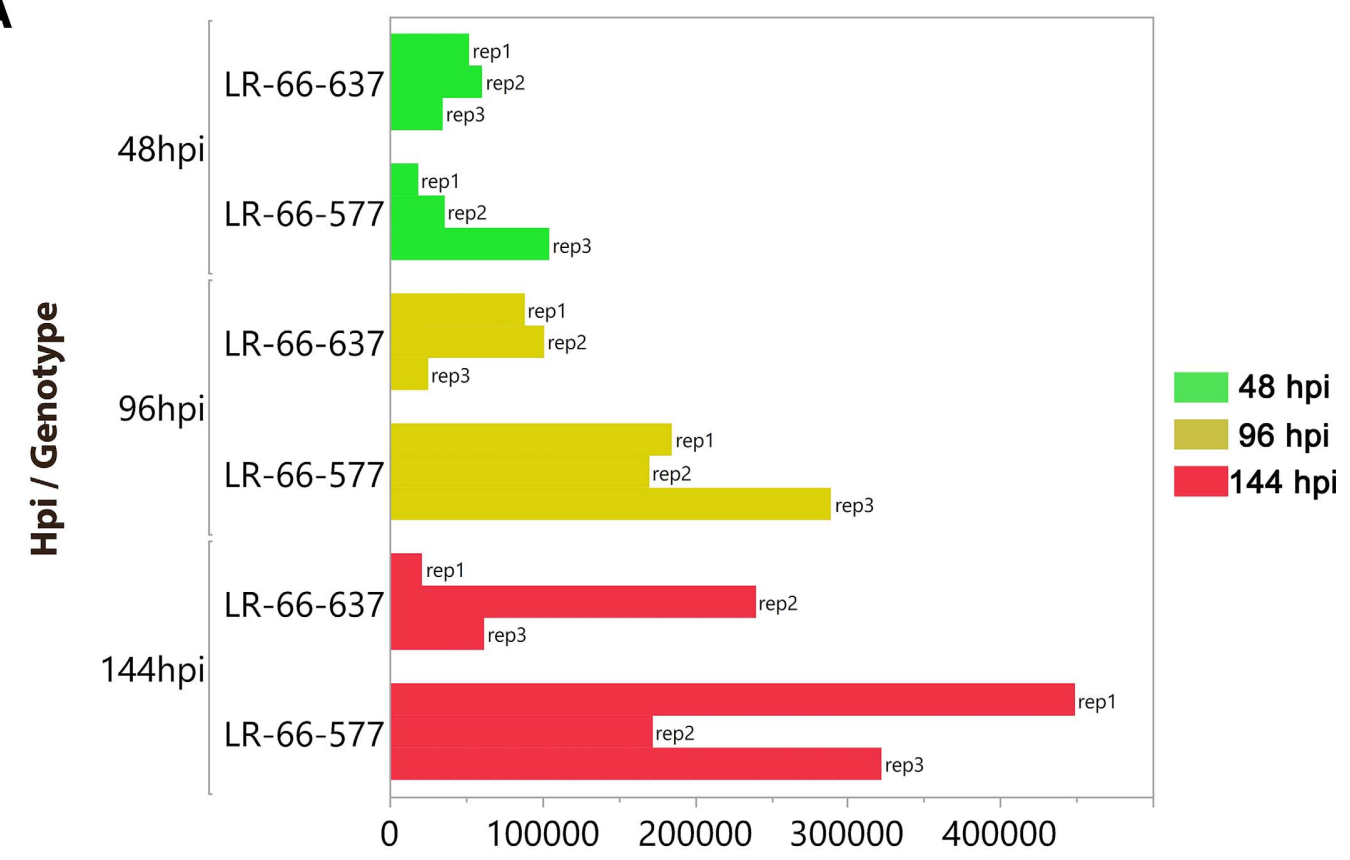

B

\section{No. of transcripts showing detectable expression}

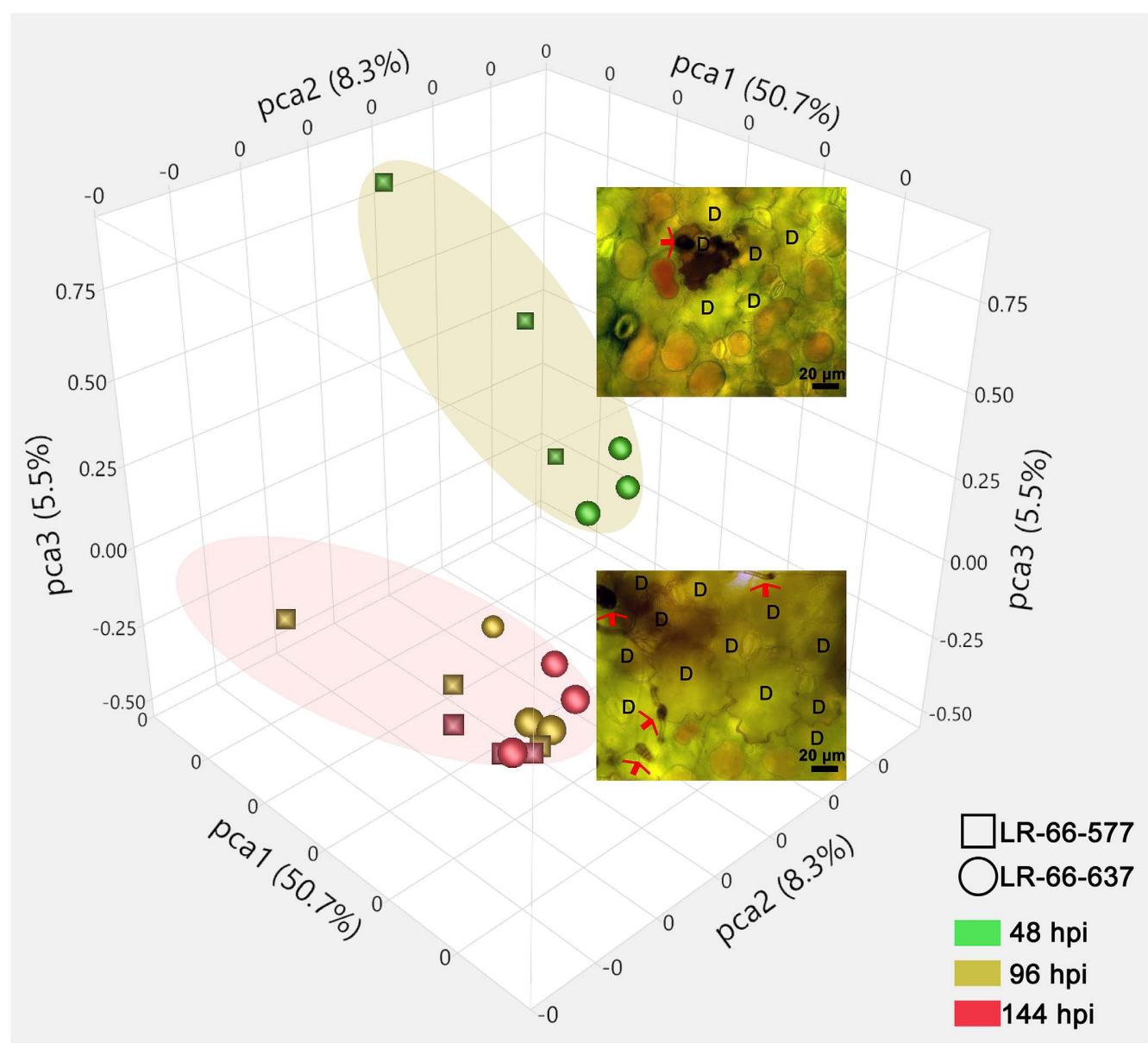

Fig. 2. Stemphylium botryosum showed temporal dynamics in gene expression during the infection process. A, Summary of total number of transcripts showing detectable expression in susceptible LR-66-577 and resistant LR-66-637 at 48, 96, and 144 h postinoculation (hpi). B, Variability assessment of S. botryosum gene expression among 18 infected Lens ervoides samples using principal component analysis (PCA). Embedded photos in the PCA plot were obtained from plant cell viability tests reflecting the most representative infection status in LR-66-577 at 48 hpi (top) and 96 hpi (bottom). Cell death in the resistant RIL LR-66-637 was also observed at these time points, though the frequency was lower compared with the susceptible RIL LR-66577 (LR-66-637 photo not shown). Red arrows point to fungal structures. Dead cells (D) were empty cells without contracted protoplasm. Photos were taken at $\times 200$ magnification. 
RILs were primarily upregulated at 96 and 144 hpi whereas, in GCN2s, genes were mainly downregulated at these two time points. To view the variation in gene composition of GCNs constructed in RILs LR-66-577 and LR-66-637, a Venn diagram was constructed, showing that 111 fungal genes of GCN1s were presented in both RILs, whereas 16 were exclusively observed in LR-66-637 and 126 in LR-66-577. For GCN2s, 42 fungal genes were commonly observed in networks of both RILs, whereas 96 were only seen in LR-66-637 and 44 in LR-66-577. These results indicated that the gene expression of $S$. botryosum in GCNs was largely dependent on the host genotypes.

To further understand the biological function of GCNs in relation to the two L. ervoides RILs, we then grouped these genes into a series of functional categories based on their annotations and the existing knowledge of gene function description in UniProt and InterPro (Fig. 6C; Supplementary Table S2). For GCN1, we found that $S$. botryosum co-upregulated a larger number of genes (genes displaying a coordinated upregulation pattern) functioning as CWDE, effector, oxidoreductase, and peptidase in the susceptible RIL LR-66-577 than in the resistant RIL LR-66-637, which was in contrast to GCN2, where the co-downregulation (genes displaying a coordinated downregulation pattern) of fungal genes functioning in those categories seemed to occur more commonly in the resistant RIL LR-66637 than in the susceptible RIL LR-66-577. These results clearly indicated that the coexpression of secretory genes in S. botryosum was shaped by the level of resistance present in the genotypes.

\section{Cross-kingdom GCNs construction \\ in L. ervoides-S. botryosum system.}

To investigate the communication between host and pathogen, we performed Spearman's rank-order correlations between 8,810 host disease-responsive genes and 1,216 fungal secretory genes separately for LR-66-577-S. botryosum and LR-66637-S. botryosum systems. High correlations $(R>0.9)$ were

Table 1. Description and completeness assessment of de novo assembled Stemphylium botryosum transcriptome

\begin{tabular}{|c|c|}
\hline Descriptive statistics & S. botryosum \\
\hline \multicolumn{2}{|l|}{ Overall assembly } \\
\hline Total trinity 'genes' & 54,027 \\
\hline Total trinity transcripts & 58,040 \\
\hline $\mathrm{GC}(\%)$ & 52.03 \\
\hline \multicolumn{2}{|l|}{ Contigs } \\
\hline Contig $\mathrm{N}_{10}$ & 3,007 \\
\hline Contig $\mathrm{N}_{20}$ & 2,195 \\
\hline Contig $\mathrm{N}_{30}$ & 1,750 \\
\hline Contig $\mathrm{N}_{40}$ & 1,374 \\
\hline Contig $\mathrm{N}_{50}$ & 1,045 \\
\hline Median contig length & 378 \\
\hline Average contig & 661 \\
\hline Total assembled bases & $38,400,522$ \\
\hline \multicolumn{2}{|l|}{ Longest isoform per gene } \\
\hline Contig $\mathrm{N}_{10}$ & 2,864 \\
\hline Contig $\mathrm{N}_{20}$ & 2,094 \\
\hline Contig $\mathrm{N}_{30}$ & 1,660 \\
\hline Contig $\mathrm{N}_{40}$ & 1,295 \\
\hline Contig $\mathrm{N}_{50}$ & 955 \\
\hline Median contig length & 366 \\
\hline Average contig & 628 \\
\hline Total assembled bases & $34,069,500$ \\
\hline \multicolumn{2}{|l|}{ Ascomycota BUSCO genes } \\
\hline Complete and single-copy BUSCOs (S) & 869 \\
\hline Complete and duplicated BUSCOs (D) & 312 \\
\hline Fragmented BUSCOs (F) & 101 \\
\hline Missing BUSCOs (M) & 33 \\
\hline Total BUSCO groups searched & 1,315 \\
\hline Total BUSCO groups retrieved $(\%)$ & 97.5 \\
\hline
\end{tabular}

observed among 5,096 genes of the LR-66-577-S. botryosum system and 5,301 genes of the LR-66-637-S. botryosum system (Supplementary Table S3). Among those genes, 3,239 genes were common to the interactome of $S$. botryosum and both RILs, whereas 1,857 genes were only observed in the LR-66577-S. botryosum system, and 2,062 in the LR-66-637-S. botryosum system (Fig. 7A). GCNs were constructed for the interactomes that were common to both RILs and those that were specific to each RIL. After excluding genes that only formed minor networks $(n<30)$ among the 3,239 genes of the transcriptome common to both $S$. botryosum-infected RILs, 3,193 genes remained (Fig. 7A). Results also showed that the coexpressions of 2,593 genes were highly similar in their topological structures and expression patterns between the two systems (Supplementary Fig. S1), which indicated that a considerable degree of similarity in host genotype-pathogen interactions occurred. Another 361 genes were involved in major GCNs specific to the LR-66-577-S. botryosum system, and 239 genes in major GCNs specific to the LR-66-637-S. botryosum system (Fig. 7A; Supplementary Table S4). In the susceptible host LR-66-577, functional categorization for these genes showed that fungal secretory genes belonging to CWDE, peptidase, oxidoreductase, and effector were co-upregulated along with host genes functioning as stress response, serine- or threonine-protein kinase, pathogenesis-related protein, oxidoreductase, and iron-related activities (Fig. 7B and C; Supplementary Table S4). Upregulation of these fungal genes seemed to coincide with the suppression of a series of host genes functioning as signal, hydrolase, DNA-binding, motor protein, monooxygenase, and microtubule. In the resistant host LR-66-637, we found that the promotion of fungal CWDE genes activated the expression of host genes functioning as glycosidase and transferase, while coinciding with the suppression of other host genes encoding membrane and metal-binding-related proteins.

For the 1,857 genes exclusive to the LR-66-577-S. botryosum system and the 2,062 genes exclusive to the LR-66-637-S. botryosum system, two GCNs (>30 genes) were constructed for each RIL (Fig. 8A and B; Supplementary Table S5). Comparison of gene expression of major GCNs between LR-66-577-S. botryosum and LR-66-637-S. botryosum systems showed opposing trends, because genes in the former were co-upregulated and genes in the latter were co-downregulated at 96 and 144 hpi. For the minor GCNs, genes in the LR-66-577-S. botryosum system were primarily co-downregulated during the infection process, whereas other genes in LR-66-637-S. botryosum were co-upregulated between 48 and $96 \mathrm{hpi}$, then co-downregulated between 96 and 144 hpi. To understand the biological function in host genotypes, we then performed functional enrichment analysis for these host genes for each of the GCNs (Fig. 8C and D). Functional enrichment analysis revealed no significant result from the minor networks involving either RIL but, for major GCNs, significant terms of "transmembrane helix", "transferase", "metal-binding", and "oxidoreductase" were observed in the LR-66-577-S. botryosum system, while "signal", "DNA-binding", "nucleus", and "transcription" were seen in the LR-66-637-S. botryosum system. To further investigate the interaction between pathogen and host, we retrieved the host genes that were significantly enriched, identified the top 10 fungal secretory genes displaying the highest hubness in the network, and constructed the interactive networks between these two sets of genes for each of RIL (Fig. 8E and F). For the susceptible host LR-66-577-S. botryosum system, host genes enriched in transmembrane helix, transferase, oxidoreductase, and metal-binding were co-upregulated with 10 fungal hub secretory genes, some of which encoded tyrosinase, glycoside hydrolase, $\alpha$ or $\beta$ hydrolase, and endoglucanase (Supplementary Table S5). From this hub gene list, three were predicted 
as effectors, including a gene encoding a CFEM domaincontaining protein and two genes without clear characterization. For the resistant host LR-66-637-S. botryosum system, host genes enriched in signal, DNA-binding, nucleus, and transcription were co-downregulated with another 10 fungal hub secretory genes, some of which were translated to glucanase, alkaline phosphatase, chitin deacetylase, and glycoside hydrolase. Three effectors were also predicted from this hub gene list, including a chitin deacetylase-like protein, a calycin-like protein, and an uncharacterized protein.

\section{DISCUSSION}

The separation of pathogen and host transcriptomes is a prerequisite for an investigation of cross-kingdom gene coexpression relationships; in this case, between a fungal pathogen and host plant. In this study, we took advantage of the L. culinaris reference genome to separate $S$. botryosum reads from the interactome and performed de novo assembly on these reads to generate an in planta $S$. botryosum transcriptome of high quality. Based on the total number of $S$. botryosum transcripts and PCA among samples, the transcription behavior of this pathogen during the early infection process (48 hpi) could be distinguished from the late infection phase (96 and $144 \mathrm{hpi}$ ), indicating a temporal in planta gene expression trend of $S$. botryosum. Host cell viability testing showed that cell death occurred at a much higher degree at 96 and 144 hpi than that at 48 hpi. We previously showed that, at 48 hpi, first chlorotic spots became visible to the naked eye, whereas first signs of necrosis appeared at $72 \mathrm{hpi}$ and fungal biomass dramatically increased after $96 \mathrm{hpi}$ (Cao et al. 2019). Taken together, this suggests that S. botryosum undergoes major gene expression reprogramming between $48 \mathrm{hpi}$ and the later infection period.

Fungal secretory proteins serve as important virulence determinants interacting with the host plant defense system, the result of which determines whether infection is successful (Lowe and Howlett 2012). In the $S$. botryosum transcriptome, 1,216 secretory proteins were successfully predicted in the present study. A series of significant GO terms of "secretory vesicle", "external encapsulating structure", "hydrolase activity", "fungal-type cell wall organization or biogenesis", and "polysaccharide catabolic process" enriched from these 1,216 genes indicated that a large number of secretory proteins in $S$. botryosum possess hydrolytic

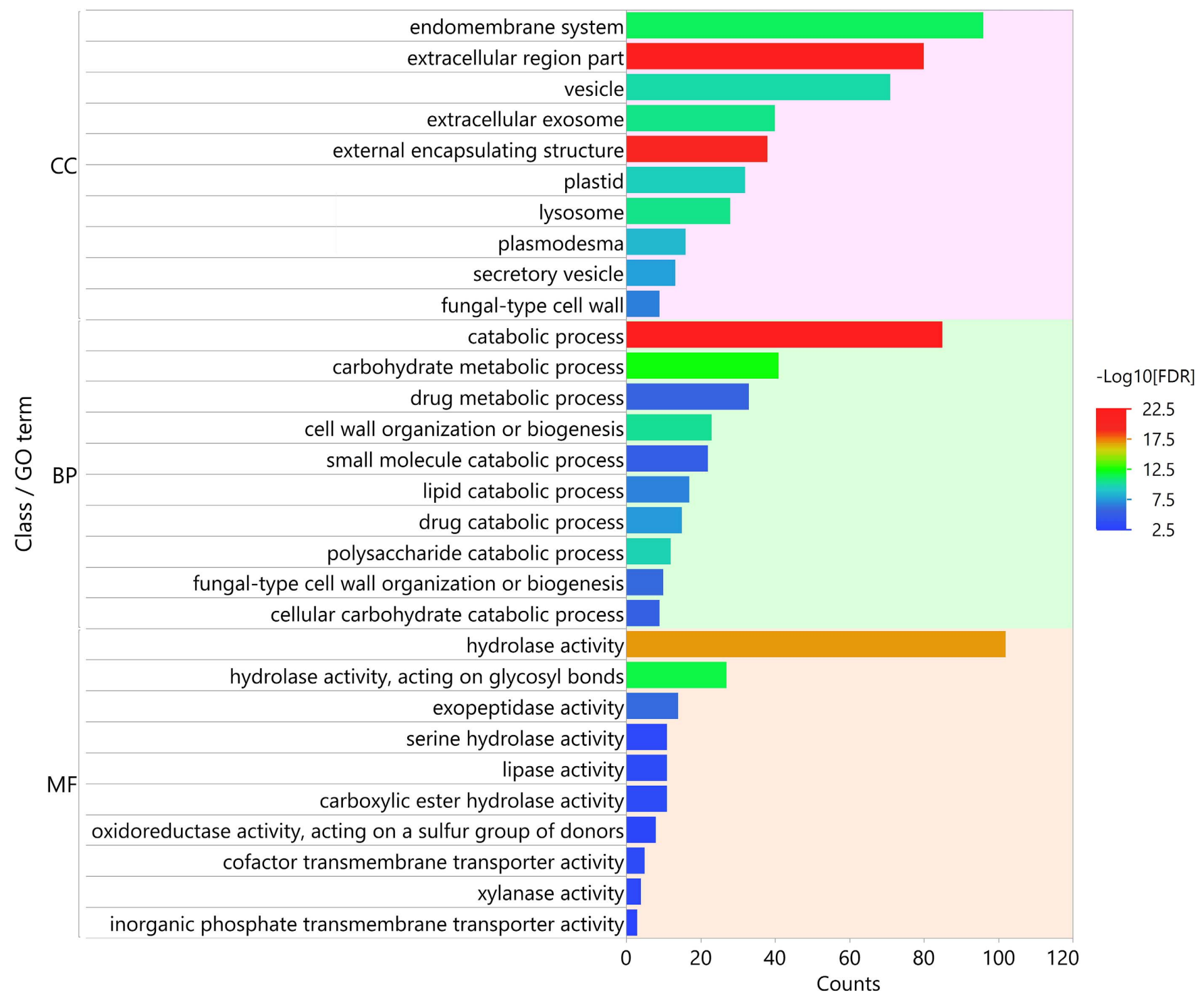

Fig. 3. Summary of gene ontology (GO) enrichment analysis for 1,216 predicted Stemphylium botryosum secretory genes. The 10 most significantly enriched GO terms were shown for each category: cellular component $(\mathrm{CC})$, biological processes (BP), and molecular function (MF). FDR $=$ false discovery rate. 
activities and may target host cell wall systems, which was expected based on research of other necrotrophs (Zeng et al. 2018). An assessment of variation in the secretory gene expression repertoire of $S$. botryosum among samples collected at different infection stages via 2D-PCA clearly demonstrated that $S$. botryosum reprogramed its secretory genes between the early and late infection stages (Fig. 4), which was similar to global transcriptional behavior of $S$. botryosum (Fig. 2B).

To date, an understanding of co-transcriptional behavior of secretory genes of pathogens has been largely elusive. Here, we found that the coexpression of fungal secretory genes was, to a large degree, influenced by the host genotype; in this case, primarily by different levels of resistance to Stemphylium blight. After infection occurred, a larger number of fungal genes encoding CWDEs, effectors, oxidoreductases, and peptidase were co-upregulated in the susceptible RIL LR-66-577 as the incubation period increased, suggesting that the promotion of secretory proteins may account for the increased susceptibility in LR-66577. Similarly, a previous study showed that the augmented virulence of Colletotrichum spp. in tomato was achieved by the overexpression of CWDEs during the necrotrophic phase (Alkan et al. 2015). Bandara et al. (2018) found that the necrotrophic pathogen Macrophomina phaseolina promoted charcoal rot susceptibility in sorghum by enhanced synthesis of a series of CWDEs. An increase in the expression of peptidases or oxidoreductases, which are important virulence factors of several necrotrophic pathogens, including B. cinerea, Rhizoctonia solani, and Sclerotinia sclerotiorum, was also associated with increases in disease severity (Foley et al. 2016; Kim et al. 2011; Zhang et al. 2019). In contrast, the colonization of Stemphylium botryosum in the resistant host LR-66-637 underwent completely different processes, in that the majority of secretory genes functioning as CWDE, oxidoreductase, and peptidase were co-downregulated as the infection period increased. Previous studies showed that the inhibition of these fungal enzymes was an important defense strategy against necrotrophic pathogens in multiple hosts (Juge 2006; Laluk and Mengiste 2010; Silva et al. 2018); hence, we hypothesize that the downregulation of these fungal secretory genes

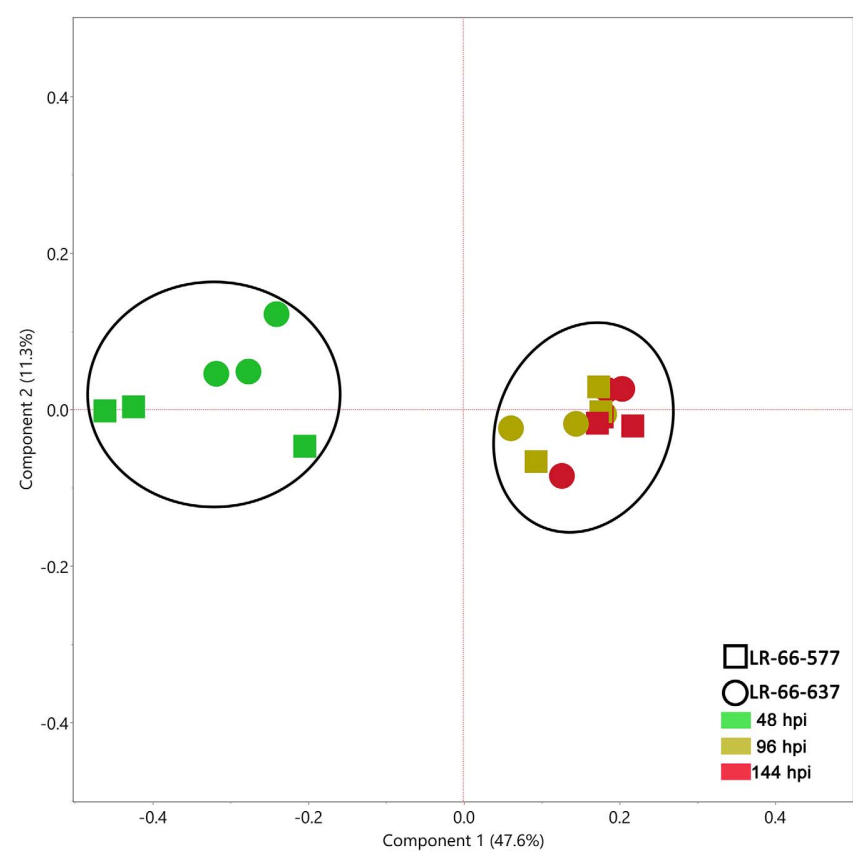

Fig. 4. Expression variability assessment of 1,216 Stemphylium botryosum secretory genes among 18 infected Lens ervoides samples; hpi = hours postinoculation. represents an important resistance mechanism in LR-66-637 that limited the invasion by $S$. botryosum.

We also constructed cross-kingdom GCNs to systemically link fungal secretory genes with host disease-responsive genes to gain insights into how these two organisms communicate with each other. For the susceptible host LR-66-577, it was shown that the co-upregulation of fungal CWDEs, peptidases, effectors, and oxidoreductases coincided with a series of host defensive responses such as redox change, serine or threonine kinase expression, and stress and disease responses. This was not observed in the resistant host LR-66-637, indicating that the plant-pathogen interaction of this kind was specific to the susceptible RIL LR-66-577 and likely accounted for its compromised disease resistance. Previous studies showed that necrotrophic pathogens can boost their virulence by employing effectors and toxins to hijack host immune systems (e.g., by activating immune responses resulting in aggravated hypersensitive response [HR] to cause cell death around infection sites) (Shi et al. 2016). This host immune response is highly effective against biotrophic organisms but facilitates the colonization of host tissues by necrotrophs. For examples, Lorang et al. (2007) reported that the overexpression of the nucleotidebinding site leucine-rich repeat $L O V I$ significantly induced cell death and exacerbated disease severity in A. thaliana infected by the necrotrophic pathogen Cochliobolus victoriae. HammondKosack and Rudd (2008) introduced a compatible interaction model for the Mycosphaerella graminicola-wheat system, explaining that the pathogen can use its secretory proteins to activate TaMPK6 in the host, which is an important member of the disease resistance signaling pathway, to initiate the HR-triggered programed cell death and plant susceptibility.

In addition, it appears that the co-upregulation of $S$. botryosum secretory genes with oxidative (tyrosinase) or hydrolytic ( $\alpha$ or $\beta$ hydrolase, glycoside hydrolase, and endoglucanase) abilities and host membrane-related genes was a signal motif for
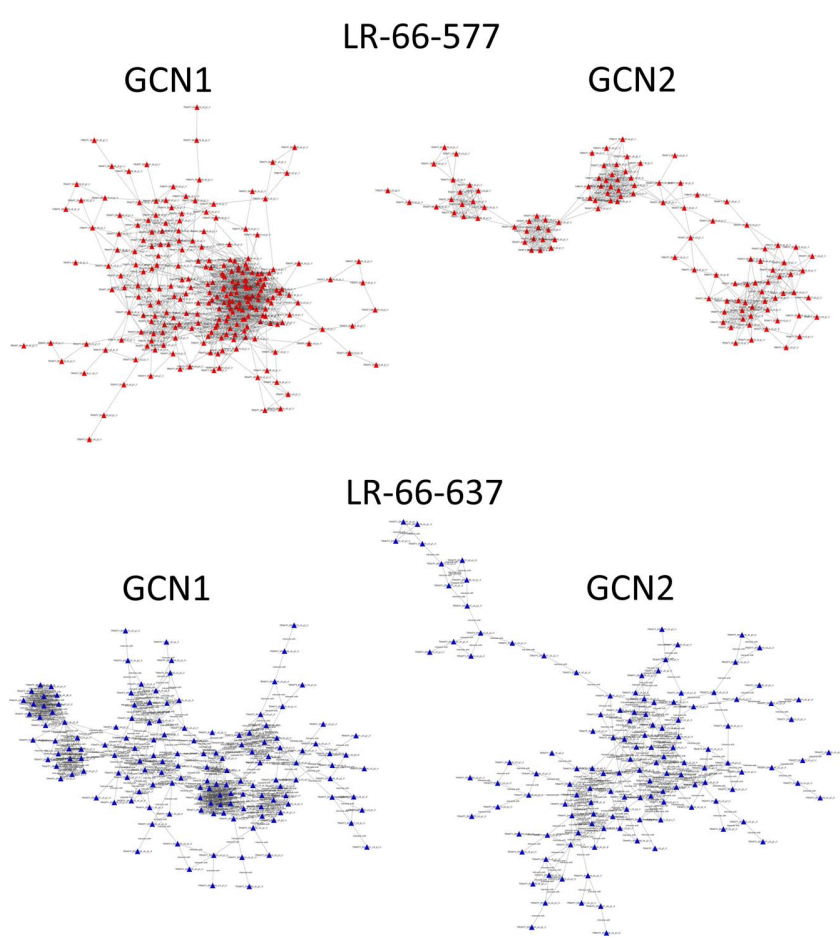

Fig. 5. Construction of gene coexpression networks (GCNs) for 1,216 Stemphylium botryosum secretory genes in two Lens ervoides hosts. Network construction was based on highly correlated gene pairs with the correlation of coefficient $>0.9$. Only major networks (size $>30$ genes) were retained. 
susceptibility because this activity was only observed in LR-66577. Thus far, studies regarding host-necrotroph interactions have mostly been focused on either the host or the pathogen. For example, activation of the membrane-anchored gene BIKI resulted in severe susceptibility in Arabidopsis upon infection with $B$. cinerea (Veronese et al. 2006). In tomato and tobacco challenged with $B$. cinerea, Cai et al. (2020) found that the overexpression of SIREM1 in the plasma membrane significantly raised reactive oxygen species in host cells, promoted cell death, and increased host susceptibility. From the pathogen angle, Zhu et al. (2017a) found that a secretory xyloglucanase of $B$. cinerea may interact with the proteins anchoring in the plant membrane

\section{A}



48 hpi 96 hpi 144 hpi

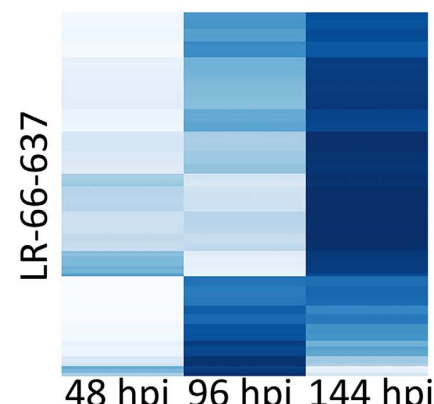

GCN2



48 hpi 96 hpi 144 hpi

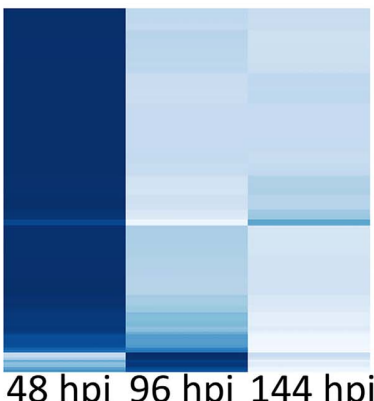

B

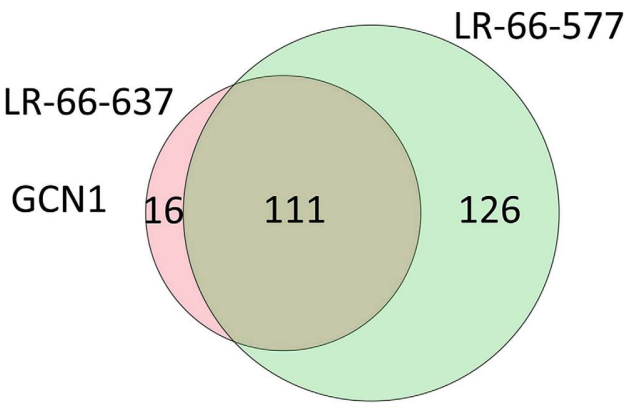

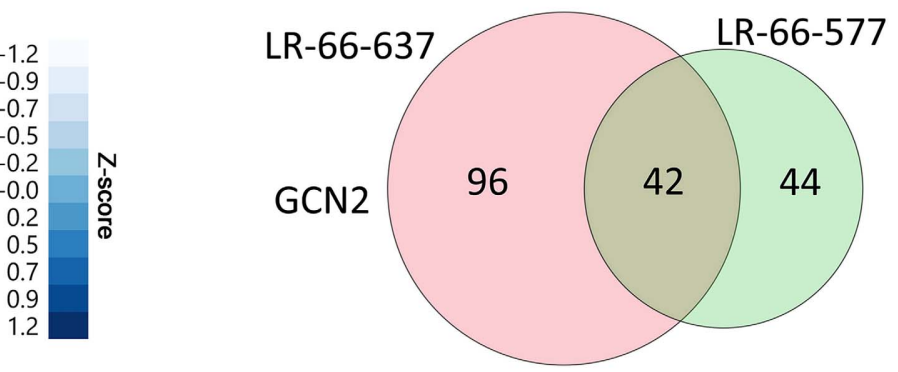

C

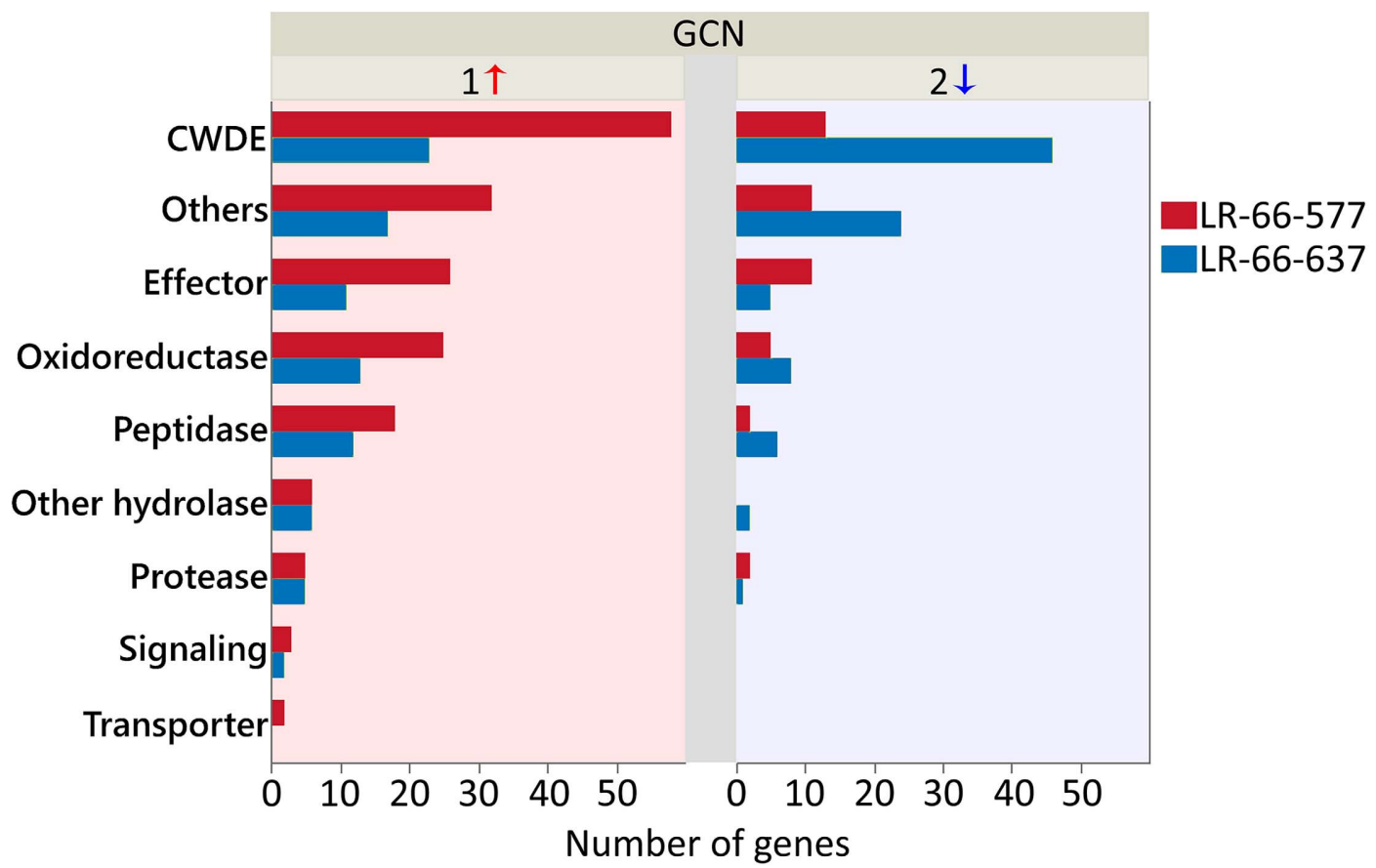

Fig. 6. Comparison of Stemphylium botryosum secretory gene coexpression networks (GCNs) between the susceptible LR-66-577 and the resistant LR-66-637. A, Relative gene expression ( $z$ score) of GCNs were displayed in heatmaps B, Variation of gene components between GCNs was displayed in Venn graphs. C, Functional categorization of secretory proteins based on their annotations and documented knowledge. CWDE $=$ cell well degradation enzyme. Arrows indicate the gene expression trend, with up and down arrows indicating upregulation and downregulation, respectively, during the infection process. 
to induce excessive cell death and susceptibility in dicot plants, including tobacco, tomato, and bean. From the perspective of both host and pathogen, Lyu et al. (2016) found that the small secreted virulence-related protein SsSSVP1 from Sclerotinia sclerotiorum particularly concentrated at the periphery of the pathogen's cell membrane and could interact with the host protein QCR8 to induce plant cell death in tobacco. However, it was not clear how SsSSVP1 crossed the plant plasma membrane and what roles plant membrane proteins played in these events. Based on this, we suspect that secretory proteins of Stemphylium botryosum may interact with membrane proteins in LR-66-577 to exacerbate disease severity. To gain an insight into these secretory genes, we identified the top 10 hub secretory genes in the LR-66-577-S. botryosum network in which these fungal genes possessed the highest level of connectivity. Among these 10 hub genes, 3 were predicted to be effectors, including 1 CFEM domain-containing gene, and the other 2 genes were without clear known function. These effectors most likely bind to host proteins. CFEM proteins contain eight conserved cysteines and are only observed in fungi. Studies indicate that these proteins are of high importance for pathogenesis in various pathogens (Zhang et al. 2015). In S. sclerotiorum and B. cinerea, CFEM proteins were also predicted as effectors, activation of which could induce leaf necrosis in hosts (Guyon et al. 2014; Zhu et al. 2017b). One recent study also indicated that CFEM effectors could accumulate in the plant membrane (Gong et al. 2020). However, it is still unknown which and how plant proteins recognize and bind with CFEM effectors. The CFEM gene possessing high hubness in the susceptible LR-66-577-S. botryosum network should be an ideal target for further investigation of its interaction with host genes, especially for those membrane-related genes that CFEM genes are highly correlated with. These may represent susceptibility genes, and could represent targets for resistance breeding.

\section{MATERIALS AND METHODS}

Plant materials, pathogen inoculation, RNA sequencing, and separation of fungal and plant sequences.

Plant and fungal materials, inoculation procedures, RNA extraction, library construction, sequencing, and the separation of plant and pathogen sequences were described in detail previously
A

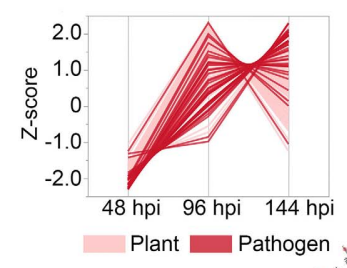

LR-66-577

(361 genes)
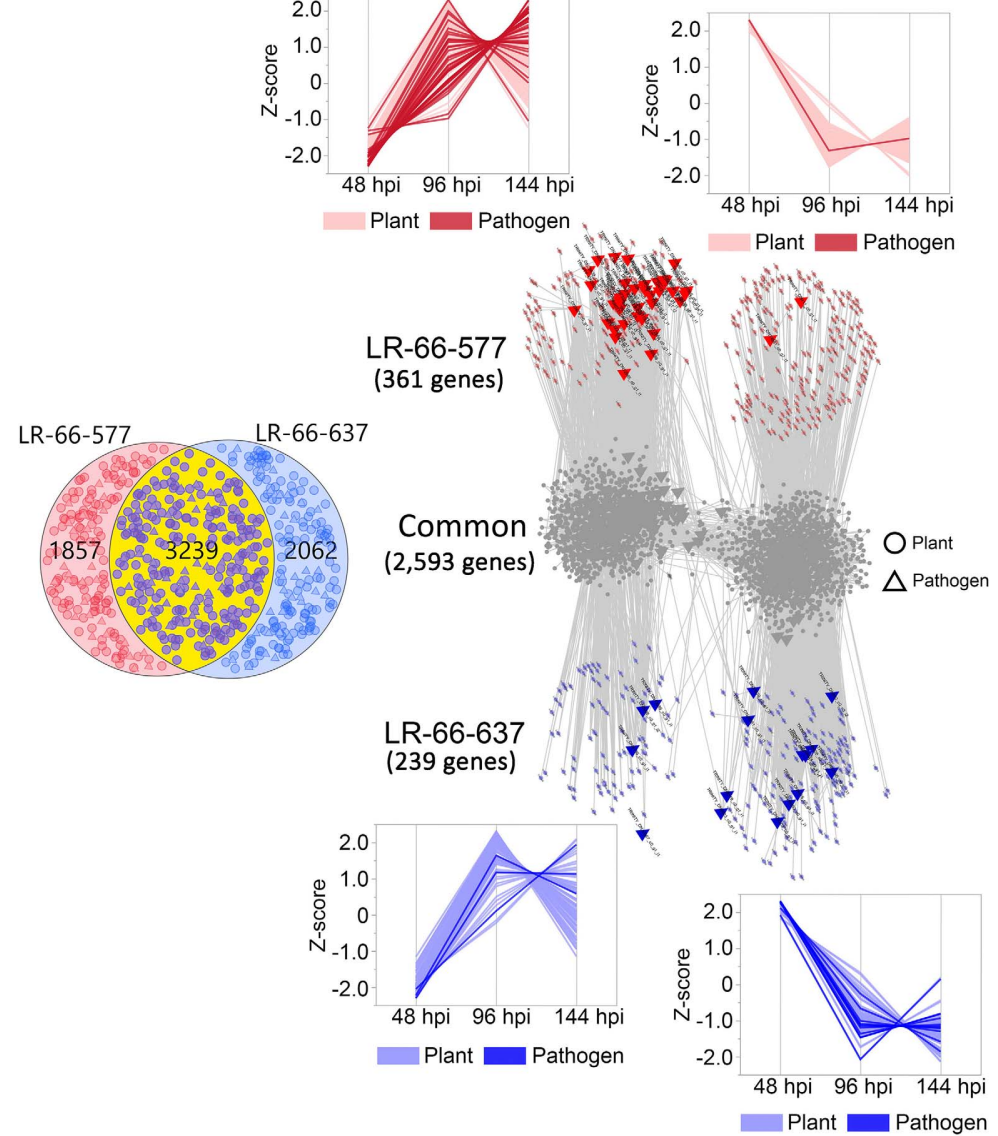

Plant Pathogen

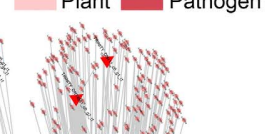

B

C

C Plant Transmembrane helix 21

Transmembrane

Membrane

Glycosidase

Transferase

Metal-binding

Signal

Hydrolase

DNA-binding

Acyltransferase

Motor protein

Monooxygenase

Microtubule

Stress response

Serine/threonine-protein kinase

Pathogenesis-related protein

Oxidoreductase

$$
\text { Iron }
$$

LR-66-577 LR-66-637
Expression

(48- 144 hpi)

up

down
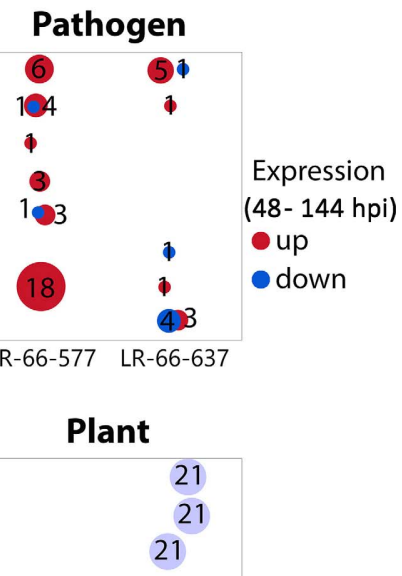

5

19

7

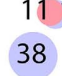

17

11

4

3

6

4

4

6

3

$11^{10}$

9
Expression

(48- 144 hpi) up

down

Fig. 7. Cross-species (Lens ervoides-Stemphylium botryosum) gene coexpression analysis for 3,239 genes displaying strong pairwise correlations $(R>0.9)$ in both recombinant inbred lines (RILs). A, Comparison of cross-kingdom networks constructed of the two RILs. Genes with strong correlations $(R>0.9)$ are depicted in the Venn diagram, with the red area indicating those specific to the LR-66-577-S. botryosum system and blue to the LR-66-637-S. botryosum system, and the yellow area containing genes with strong correlations $(R>0.9)$ in both system. To visualize the variation of gene correlation topology of these 3,239 genes overlapping between the two systems, gene expression networks constructed for the two systems were compared. Only major networks were retained (size > 30). Gray nodes (2,593 genes) were elements of major networks shared by both $L$. ervoides $-S$. botryosum systems. The top red highlighted (361 genes) and the bottom blue highlighted (239 genes) nodes were those genes that were specifically connected to major gene coexpression networks (GCNs) of the LR-66-577-S. botryosum and LR-66-637-S. botryosum systems, respectively. Expression trends of these coexpressed genes were shown in the adjacent line graphs; hpi = hours postinoculation. B and $\mathbf{C}$, Functional categorization of fungal and host coexpressed genes. Functional categorization of fungal genes was conducted manually based on documented knowledge. CWDE $=$ cell wall degradation enzyme. Host gene functional enrichment was performed via the DAVID online website using a cut-off $P$ value of 0.05 to declare the significant enrichment. 
A

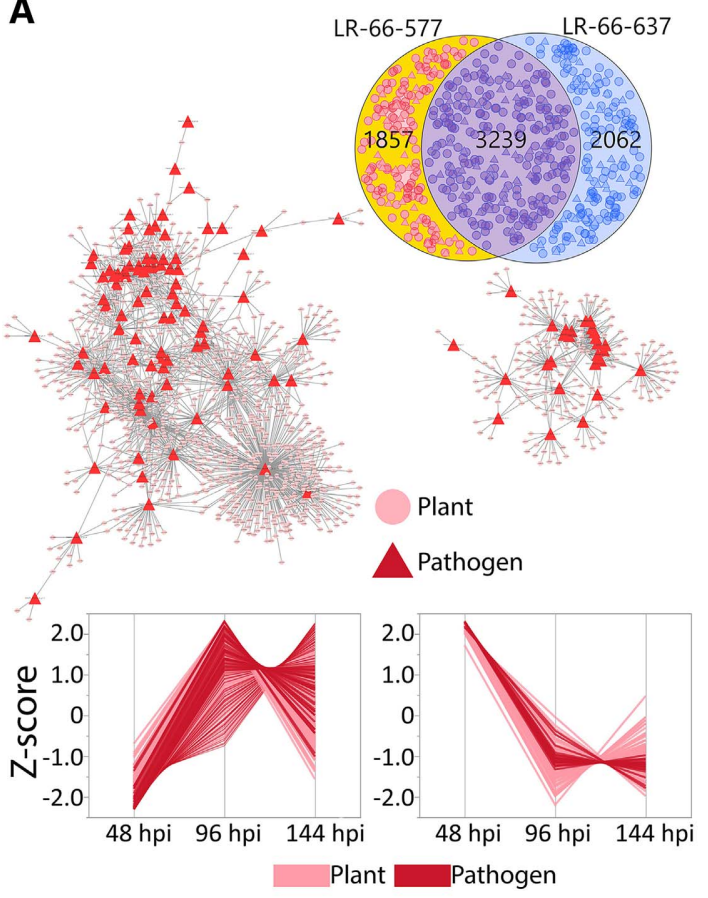

C

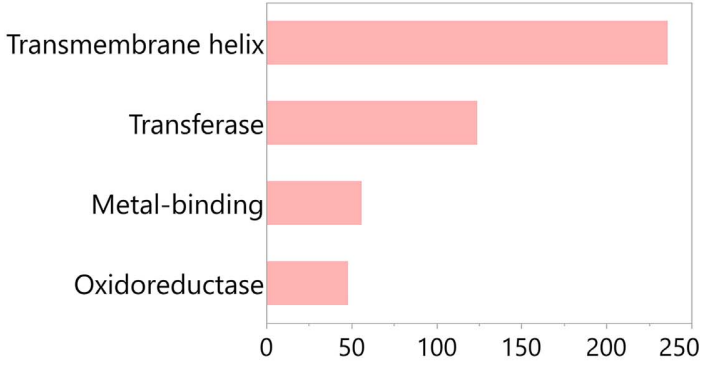

E

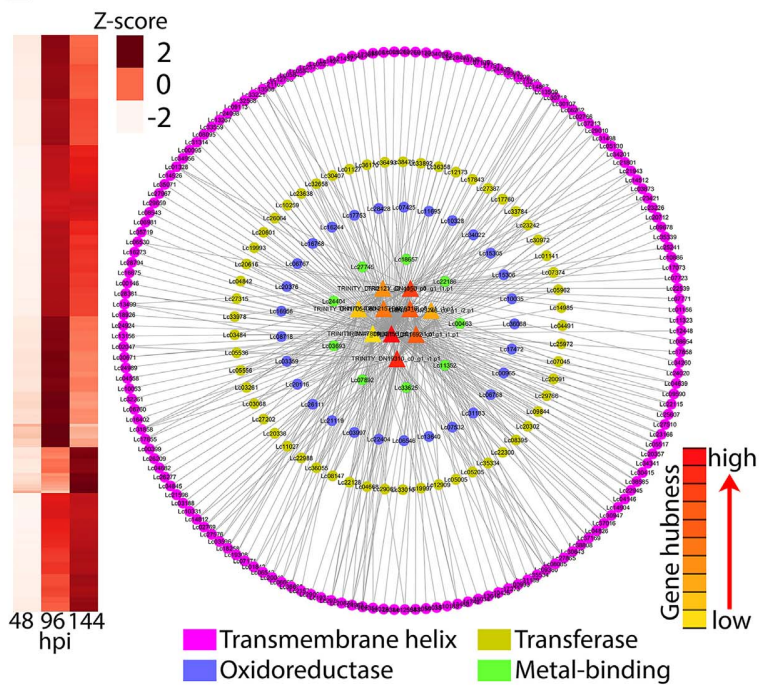

B
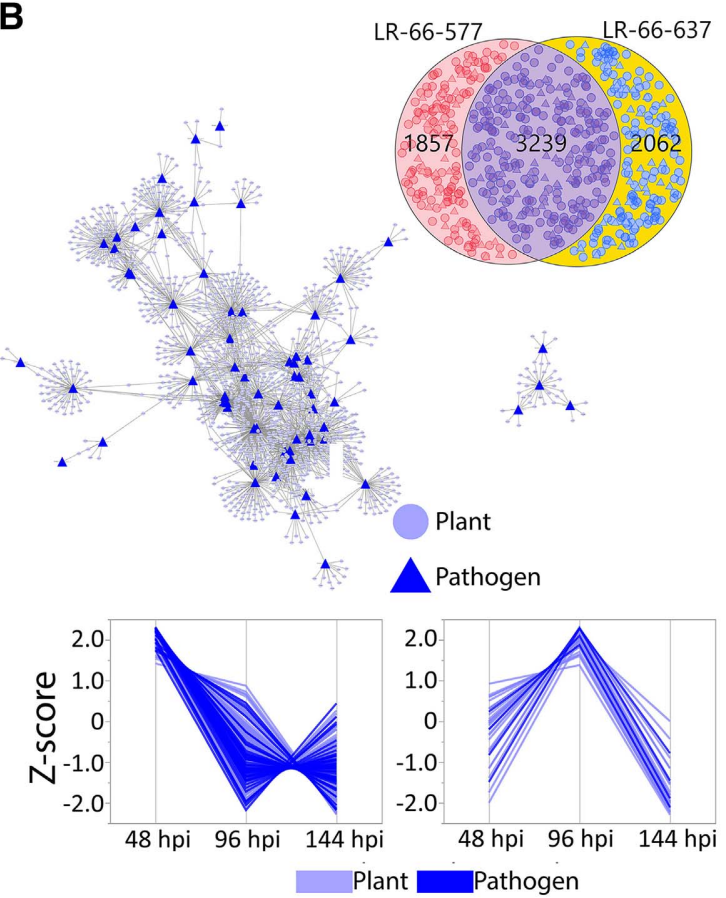

D

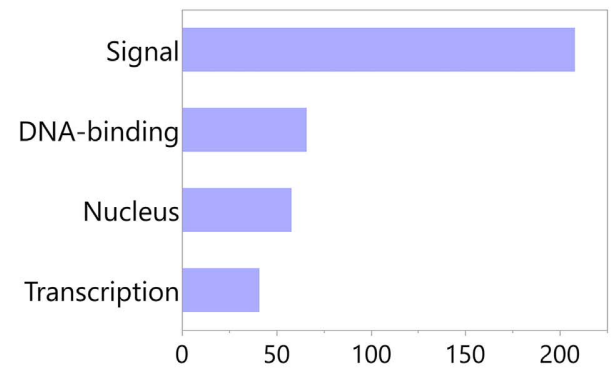

$\mathbf{F}$

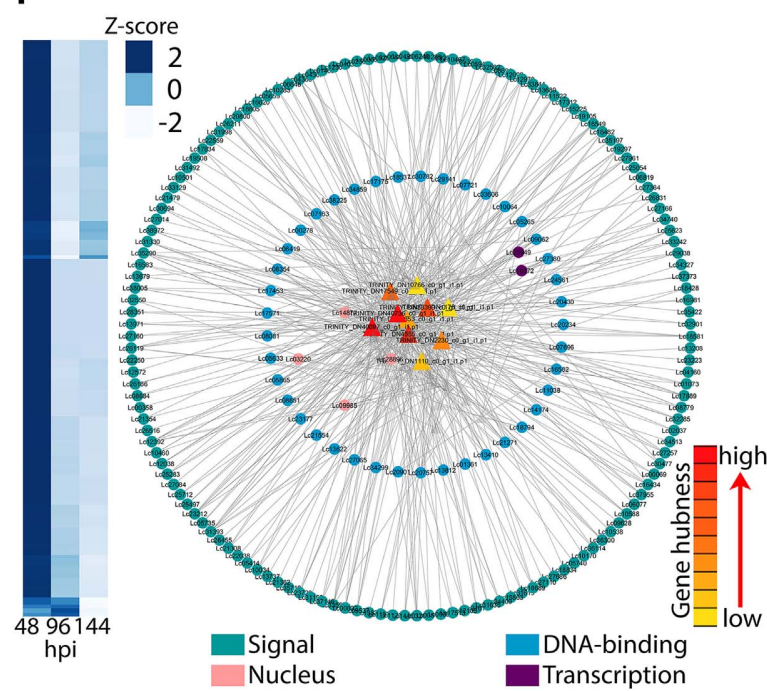

Fig. 8. Lens ervoides-Stemphylium botryosum gene coexpression analysis for 1,857 in the LR-66-577-S. botryosum and 2,062 genes in the LR-66$637-S$. botryosum systems, only displaying strong gene correlation $(R>0.9)$. Construction of gene coexpression networks (GCNs) in the A, LR-66$577-S$. botryosum and B, LR-66-637-S. botryosum systems; hpi = hours postinoculation. The yellow highlighted area in the Venn diagram contained genes that formed strong correlation $(R>0.9)$ in either LR-66-577 or LR-66-637. Gene expression trends for GCNs were displayed by line graphs shown below. Host gene functional enrichment in C, LR-66-577 and D, LR-66-637. Host gene functional enrichment was performed via the DAVID online website, with a cut-off $P$ value of 0.05 to declare the significant enrichment. Construction of GCNs using significantly enriched host genes and top 10 fungal secretory genes with the highest hubness in E, LR-66-577 and F, LR-66-637. Gene expression trends of GCNs were displayed by heatmaps. 
by Cao et al. (2019). Briefly, transgressive segregant RIL LR-66637 (partially resistant to S. botryosum) and RIL LR-66-577 (susceptible to $S$. botryosum) were selected from an $\mathrm{F}_{9}$ interspecific population derived from the cross between $L$. ervoides accessions L01-827A and IG72815 (Bhadauria et al. 2017). Four-week-old seedlings (four plants in each of three replicate pots for each RIL and three sampling time points of 48, 96, and 144 hpi, determined based on histopathological examination and fungal biomass quantification) were inoculated with approximately 2 to 2.5 ml per plant of a $1 \times 10^{5}$ conidia $\mathrm{ml}^{-1}$ suspension of $S$. botryosum isolate SB19. Total RNA was extracted from frozen leaves using the RNeasy Plant Mini Kit (Qiagen Company, Hilden, Germany) following the manufacturer's instructions, and high-quality samples (RNA integrity number $>7$ ) were used for library construction using the TruSeq Stranded Total RNA kit (Illumina, Inc., San Diego, CA, U.S.A.). The constructed libraries were then fed into an Illumina HiSeq 2500 sequencer using TruSeq SBS KITHS V4 (Illumina) for paired-ended sequencing with a read length of 125 bp at Canada's Michael Smith Genome Sciences Center (BCGSC, Vancouver, BC, Canada).

The returned raw reads were first loaded into Trimmomatic (version 0.36) software (Bolger et al. 2014) to remove lowquality reads, and sequencing adaptors and high-quality reads were mapped against the $L$. culinaris genome V1.2 in STAR 2.6.1a (default settings) (Dobin et al. 2013). Successfully mapped reads were recognized as common reads shared between $L$. culinaris and L. ervoides. For pathogen-inoculated samples at 48, 96, and $144 \mathrm{hpi}$, unmapped reads were considered to be from $S$. botryosum or L. ervoides (L. ervoides-specific reads). For noninoculated control samples, unmapped reads were from L. ervoides (L. ervoides-specific reads); therefore, to recover $S$. botryosum reads, unmapped reads from noninoculated control samples were de novo assembled (Trinity version 2.84, default settings) into a "leftover $L$. ervoides transcriptome" which was used as template for alignment of unmapped reads from pathogen-inoculated samples (Grabherr et al. 2011). After excluding reads that were successfully aligned to the leftover $L$. ervoides transcriptome, the remaining unaligned reads were matched against the L. culinaris genome with Fastq_screen (version 0.11.4) (Wingett and Andrews 2018). Only unmapped and unmatched reads were recognized as $S$. botryosum reads for de novo transcriptome assembly.

\section{De novo assembly, quantification, and annotation of $S$. botryosum transcriptome.}

The enriched $S$. botryosum reads from all samples were de novo assembled in Trinity using the default settings. The general statistics of the assembled $S$. botryosum transcriptome was assessed with the script "TrinityStats.pl" provided by Trinity. To evaluate the transcriptome completeness, we used BUSCO to compare all $S$. botryosum transcripts with the BUSCO content of the Ascomycota division to which S. botryosum belongs (Seppey et al. 2019). The script "align_and_estimate_abundance.pl" affiliated with Trinity was used to assess the gene expression for all samples of $S$. botryosum in the form of raw counts and TPM. The expression form of TPM was mainly used for the subsequent analyses, including PCA and gene coexpression analyses.

The software CD-hit (version 4.81) (Fu et al. 2012) was used to remove redundant reads of the transcriptome, before ORFs with at least 100 amino acids were identified from the transcriptome with TransDecoder (version 5.5.0, default settings) (Haas et al. 2013). These ORFs were functionally annotated by BlastP (e-value $=1 \mathrm{e}-5$ ) against the fungi RefSeq database in Diamond (version 0.9.8) (Buchfink et al. 2015) and hmmScan against Pfam database in HMMER (version 3.1b2) (Finn et al. 2015). The coding sequences (CDS) prediction of ORFs was conducted in TransDecoder, with the setting of retaining RefSeq or Pfam homologs even if their CDS were not successfully predicted.

\section{Secretome prediction.}

For secretome prediction, predicted proteins were submitted to SignalP (version 3.0) (Bendtsen et al. 2004) for signal peptide, to TargetP (version 1.1, location $=$ Secreted) $($ Emanuelsson et al. 2007) for location, and to TMHMM (version 2.0) (Krogh et al. 2001) for transmembrane domain predictions. All analyses using these softwares followed default settings. Only proteins potentially carrying signal peptides but lacking transmembrane domains were recognized as signal proteins. In addition, proteins were scanned for the C-terminal motif of HDEL or KDEL, because these motifs are knowns as ER-retention signals. Only signal proteins without either of these motifs were recognized as putative $S$. botryosum secretory proteins. Furthermore, secretory proteins were annotated with InterProScan software using a series of databases (PANTHER, Pfam, Coils, Gene3D, SUPERFAMILY, SMART, PIRSF, and PRINTS) and BlastP (e-value = 1e-5) against the fungi RefSeq database (Jones et al. 2014). The effector prediction among secretory proteins was conducted using the online software EffectorP. To gain an overview of the function of these secretory proteins, the GO overrepresentation analysis was implemented by testing Pfam domains of secretory proteins against all annotatable domains (default setting) via an online DcGO website.

\section{Host viability testing.}

The viability of epidermal cells of LR-66-637 and LR-66-577 was assessed as suggested by Sari et al. (2017), with the following modifications. Before staining, forceps were used to carefully remove the first layer of abaxial epidermis cells of leaves, which promoted the penetration of the staining solution entering the inner cells of the leaf tissue without causing cell death in the mesophyll or the adaxial epidermis. The trimmed leaves were then immersed in Neutral Red salt solution $\left(0.85 \mathrm{M} \mathrm{KNO}_{3}\right.$ and 0.01 Neutral Red) to induce plasmolysis and plant cell staining. To accelerate the staining process, plant leaves containing Neutral Red were vacuum infiltrated for 5 to $10 \mathrm{~min}$, then incubated at room temperature for at least $3 \mathrm{~h}$. The adaxial epidermal cells of prepared leaves were examined with a Zeiss Axioskop 40 microscope (Carl Zeiss, Göttingen, Germany). Photos were taken using a Pixelink A686C camera (Pixelink, Rochester, NY, U.S.A.) and Zeiss Axiovision software (Carl Zeiss).

\section{Construction of in planta $S$. botryosum GCNs.}

In planta fungal GCNs based on $S$. botryosum secretory genes were constructed for each individual $L$. ervoides RIL. To do so, lists of secretory genes were first curated by removing genes with missing expression data; during the gene expression estimation process, when paired-end reads were aligned back with transcriptome, some read pairs locating in different contigs causing ambiguities in gene counting were regarded as missing expression and scored as 0 in the form of TPM. Curated genes were submitted for pairwise correlation analysis based on Spearman's rank-order correlation analysis in JMP Genomics 9.0. The correlation coefficient of 0.9 was chosen as a cut-off value to identify highly connected gene pairs for GCNs construction and visualization in Cytoscape 3.71 (Shannon et al. 2003). Of the GCNs constructed for each RIL, only major GCNs (size > 30 genes) were selected for further analysis. Overlaps of genes between GCNs with similar expression patterns between the two RILs were shown in Venn diagrams. To understand the biological roles of these secretory genes, we then manually grouped genes into a series of functional categories based on documented knowledge of gene function description in UniProt and InterPro databases. 


\section{Construction of cross-kingdom GCN}

\section{between $S$. botryosum secretory genes and}

L. ervoides disease-responsive genes.

Our previous study using the same plant-pathogen system identified 8,810 host disease-responsive genes, which were either significantly upregulated or downregulated after $S$. botryosum infection (Cao et al. 2019). To develop the interactive $S$. botryosum $-L$. ervoides GCNs, these 8,810 diseaseresponsive genes and the predicted fungal secretory genes were submitted for pairwise correlation analysis in JMP genomics 9.0. The correlation coefficient cut-off was set as 0.9 . We then performed network visualization and comparison of these correlated genes in Cytoscape to visualize the variation of gene correlation topology. Simply explained, a correlation such as gene A-gene B-gene $\mathrm{C}$-gene D could form in one system but may occur in a different form as gene $\mathrm{A}-$ gene $\mathrm{C}$ and gene $\mathrm{B}-$ gene $\mathrm{D}$ (gene $\mathrm{B}$ and gene $\mathrm{C}$ are not correlated) in the other system. When performing gene network construction, the first correlation of gene A-gene B-gene $\mathrm{C}-$ gene $\mathrm{D}$ will be regarded as one gene network with four elements but the second version of gene $\mathrm{A}$-gene $\mathrm{C}$ and gene $\mathrm{B}-$ gene $\mathrm{D}$ will be considered as two gene networks with two elements per network. Only major networks (size > 30) were used for further analysis. The gene functional enrichment analysis of host genes was performed by testing host genes against all annotated genes in the lentil genome using Medicago truncatula orthologs via the DAVID online platform. The cut-off of $P$ value $<0.05$ was used to declare a significant enrichment. For fungal genes, we manually grouped $S$. botryosum secretory genes into a series of functional categories based on the existing knowledge for the better overview of their biological roles.

\section{AUTHOR-RECOMMENDED INTERNET RESOURCES}

DAVID online platform: https://david.ncifcrf.gov

DcGO website:

https://supfam.org/SUPERFAMILY/cgi-bin/dcen- richment.cgi

EffectorP: http://effectorp.csiro.au

\section{LITERATURE CITED}

Alkan, N., Friedlander, G., Ment, D., Prusky, D., and Fluhr, R. 2015. Simultaneous transcriptome analysis of Colletotrichum gloeosporioides and tomato fruit pathosystem reveals novel fungal pathogenicity and fruit defense strategies. New Phytol. 205:801-815.

Aveling, T. A. S., and Snyman, H. G. 1993. Infection studies of Stemphylium vesicarium on onion leaves. Mycol. Res. 97:984-988.

Bandara, Y. M. A. Y., Weerasooriya, D. K., Liu, S., and Little, C. R. 2018. The necrotrophic fungus Macrophomina phaseolina promotes charcoal rot susceptibility in grain sorghum through induced host cellwall-degrading enzymes. Phytopathology 108:948-956.

Bendtsen, J. D., Nielsen, H., von Heijne, G., and Brunak, S. 2004. Improved prediction of signal peptides: SignalP 3.0. J. Mol. Biol. 340: 783-795.

Bhadauria, V., Ramsay, L., Bett, K. E., and Banniza, S. 2017. QTL mapping reveals genetic determinants of fungal disease resistance in the wild lentil species Lens ervoides. Sci. Rep. 7:3231.

Bolger, A. M., Lohse, M., and Usadel, B. 2014. Trimmomatic: A flexible trimmer for Illumina sequence data. Bioinformatics 30:2114-2120.

Borges, O. L., Stanford, H. E., and Webster, R. K. 1976. The hostpathogen interaction of alfalfa and Stemphylium botryosum. Phytopathology 66:749-752.

Buchfink, B., Xie, C., and Huson, D. H. 2015. Fast and sensitive protein alignment using DIAMOND. Nat. Methods 12:59-60.

Cai, J., Chen, T., Wang, Y., Qin, G., and Tian, S. 2020. SIREM1 triggers cell death by activating an oxidative burst and other regulators. Plant Physiol. 183:717-732.

Cao, Z., Li, L., Kapoor, K., and Banniza, S. 2019. Using a transcriptome sequencing approach to explore candidate resistance genes against Stemphylium blight in the wild lentil species Lens ervoides. BMC Plant Biol. 19:399.
Dobin, A., Davis, C. A., Schlesinger, F., Drenkow, J., Zaleski, C., Jha, S., Batut, P., Chaisson, M., and Gingeras, T. R. 2013. STAR: Ultrafast universal RNA-seq aligner. Bioinformatics 29:15-21.

Emanuelsson, O., Brunak, S., von Heijne, G., and Nielsen, H. 2007. Locating proteins in the cell using TargetP, SignalP and related tools. Nat. Protoc. 2:953-971.

Evangelisti, E., Gogleva, A., Hainaux, T., Doumane, M., Tulin, F., Quan, C., Yunusov, T., Floch, K., and Schornack, S. 2017. Time-resolved dual transcriptomics reveal early induced Nicotiana benthamiana root genes and conserved infection-promoting Phytophthora palmivora effectors. BMC Biol. 15:39.

Feng, B. Z., Zhu, X. P., Fu, L., Lv, R. F., Storey, D., Tooley, P., and Zhang, X. G. 2014. Characterization of necrosis-inducing NLP proteins in Phytophthora capsici. BMC Plant Biol. 14:126.

Figaj, D., Ambroziak, P., Przepiora, T., and Skorko-Glonek, J. 2019. The role of proteases in the virulence of plant pathogenic bacteria. Int. J. Mol. Sci. 20:672.

Finn, R. D., Clements, J., Arndt, W., Miller, B. L., Wheeler, T. J., Schreiber, F., Bateman, A., and Eddy, S. R. 2015. HMMER web server: 2015 Update. Nucleic Acids Res. 43:W30-W38.

Foley, R. C., Kidd, B. N., Hane, J. K., Anderson, J. P., and Singh, K. B. 2016. Reactive oxygen species play a role in the infection of the necrotrophic fungi, Rhizoctonia solani in wheat. PLoS One 11 e0152548.

Fu, L., Niu, B., Zhu, Z., Wu, S., and Li, W. 2012. CD-HIT: Accelerated for clustering the next-generation sequencing data. Bioinformatics 28 : 3150-3152.

Gong, A., Jing, Z., Zhang, K., Tan, Q., Wang, G., and Liu, W. 2020 Bioinformatic analysis and functional characterization of the CFEM proteins in maize anthracnose fungus Colletotrichum graminicola. J. Integr. Agric. 19:541-550.

González-Fernández, R., Valero-Galván, J., Gómez-Gálvez, F. J., and Jorrín-Novo, J. V. 2015. Unraveling the in vitro secretome of the phytopathogen Botrytis cinerea to understand the interaction with its hosts. Front. Plant Sci. 6:839.

Grabherr, M. G., Haas, B. J., Yassour, M., Levin, J. Z., Thompson, D. A., Amit, I., Adiconis, X., Fan, L., Raychowdhury, R., Zeng, Q. Chen, Z., Mauceli, E., Hacohen, N., Gnirke, A., Rhind, N., di Palma, F., Birren, B. W., Nusbaum, C., Lindblad-Toh, K., Friedman, N., and Regev, A 2011. Full-length transcriptome assembly from RNA-Seq data without a reference genome. Nat. Biotechnol. 29:644-652.

Graham, J. H. 1957. A Stemphylium disease on ladino white clover. Phytopathology 47:213-215.

Guyon, K., Balagué, C., Roby, D., and Raffaele, S. 2014. Secretome analysis reveals effector candidates associated with broad host range necrotrophy in the fungal plant pathogen Sclerotinia sclerotiorum. BMC Genomics 15:336.

Haas, B. J., Papanicolaou, A., Yassour, M., Grabherr, M., Blood, P. D., Bowden, J., Couger, M. B., Eccles, D., Li, B., Lieber, M., MacManes, M. D., Ott, M., Orvis, J., Pochet, N., Strozzi, F., Weeks, N., Westerman, R., William, T., Dewey, C. N., Henschel, R., LeDuc, R. D., Friedman, N., and Regev, A. 2013. De novo transcript sequence reconstruction from RNA-seq using the Trinity platform for reference generation and analysis. Nat. Protoc. 8:1494-1512.

Hammond-Kosack, K. E., and Rudd, J. J. 2008. Plant resistance signalling hijacked by a necrotrophic fungal pathogen. Plant Signal. Behav. 3:993-995.

Heard, S., Brown, N. A., and Hammond-Kosack, K. 2015. An interspecies comparative analysis of the predicted secretomes of the necrotrophic plant pathogens Sclerotinia sclerotiorum and Botrytis cinerea. PLoS One 10:e0130534.

Jing, L., Guo, D., Hu, W., and Niu, X. 2017. The prediction of a pathogenesis-related secretome of Puccinia helianthi through highthroughput transcriptome analysis. BMC Bioinformatics 18:166.

Jones, P., Binns, D., Chang, H. Y., Fraser, M., Li, W., McAnulla, C., McWilliam, H., Maslen, J., Mitchell, A., Nuka, G., Pesseat, S., Quinn, A. F., Sangrador-Vegas, A., Scheremetjew, M., Yong, S. Y., Lopez, R., and Hunter, S. 2014. InterProScan 5: Genome-scale protein function classification. Bioinformatics 30:1236-1240.

Juge, N. 2006. Plant protein inhibitors of cell wall degrading enzymes. Trends Plant Sci. 11:359-367.

Kim, H. J., Chen, C., Kabbage, M., and Dickman, M. B. 2011. Identification and characterization of Sclerotinia sclerotiorum NADPH oxidases. Appl. Environ. Microbiol. 77:7721-7729.

Koike, S. T., Henderson, D. M., and Butler, E. E. 2001. Leaf spot disease of spinach in California caused by Stemphylium botryosum. Plant Dis. 85:126-130. 
Krogh, A., Larsson, B., von Heijne, G., and Sonnhammer, E. L. 2001. Predicting transmembrane protein topology with a hidden Markov model: Application to complete genomes. J. Mol. Biol. 305:567-580.

Laluk, K., and Mengiste, T. 2010. Necrotroph attacks on plants: Wanton destruction or covert extortion? Arabidopsis Book 8:e0136.

Lorang, J. M., Sweat, T. A., and Wolpert, T. J. 2007. Plant disease susceptibility conferred by a "resistance" gene. Proc. Natl. Acad. Sci. U.S.A. 104:14861-14866.

Lowe, R. G., and Howlett, B. J. 2012. Indifferent, affectionate, or deceitful: Lifestyles and secretomes of fungi. PLoS Pathog. 8:e1002515.

Lyu, X., Shen, C., Fu, Y., Xie, J., Jiang, D., Li, G., and Cheng, J. 2016. A small secreted virulence-related protein is essential for the necrotrophic interactions of Sclerotinia sclerotiorum with its host plants. PLoS Pathog. 12:e1005435.

Meinhardt, L. W., Costa, G. G., Thomazella, D. P., Teixeira, P. J., Carazzolle, M. F., Schuster, S. C., Carlson, J. E., Guiltinan, M. J., Mieczkowski, P., Farmer, A., Ramaraj, T., Crozier, J., Davis, R. E., Shao, J., Melnick, R. L., Pereira, G. A., and Bailey, B. A. 2014. Genome and secretome analysis of the hemibiotrophic fungal pathogen, Moniliophthora roreri, which causes frosty pod rot disease of cacao: Mechanisms of the biotrophic and necrotrophic phases. BMC Genomics 15:164.

Morais do Amaral, A., Antoniw, J., Rudd, J. J., and Hammond-Kosack, K. E. 2012. Defining the predicted protein secretome of the fungal wheat leaf pathogen Mycosphaerella graminicola. PLoS One 7:e49904.

Mwakutuya, E., and Banniza, S. 2010. Influence of temperature and wetness periods on the development of Stemphylium blight on lentil. Plant Dis. 94:1219-1224.

Neu, E., and Debener, T. 2019. Prediction of the Diplocarpon rosae secretome reveals candidate genes for effectors and virulence factors. Fungal Biol. 123:231-239.

Pandey, V., Singh, M., Pandey, D., Marla, S., and Kumar, A. 2018. Secretome analysis identifies potential pathogenicity/virulence factors of Tilletia indica, a quarantined fungal pathogen inciting karnal bunt disease in wheat. Proteomics 18:1700473.

Sari, E., Bhadauria, V., Vandenberg, A., and Banniza, S. 2017. Genotype-dependent interaction of lentil lines with Ascochyta lentis. Front. Plant Sci. 8:764.

Seppey, M., Manni, M., and Zdobnov, E. M. 2019. BUSCO: Assessing genome assembly and annotation completeness. Methods Mol. Biol. 1962:227-245.
Shannon, P., Markiel, A., Ozier, O., Baliga, N. S., Wang, J. T., Ramage, D., Amin, N., Schwikowski, B., and Ideker, T. 2003. Cytoscape: A software environment for integrated models of biomolecular interaction networks. Genome Res. 13:2498-2504.

Shi, G., Zhang, Z., Friesen, T. L., Raats, D., Fahima, T., Brueggeman, R. S., Lu, S., Trick, H. N., Liu, Z., Chao, W., Frenkel, Z., Xu, S. S. Rasmussen, J. B., and Faris, J. D. 2016. The hijacking of a receptor kinase-driven pathway by a wheat fungal pathogen leads to disease. Sci. Adv. 2:e1600822.

Silva, M. S., Arraes, F. B. M., Campos, M. A., Grossi-de-Sa, M., Fernandez, D., Cândido, E. S., Cardoso, M. H., Franco, O. L., and Grossi-de-Sa, M. F. 2018. Review: Potential biotechnological assets related to plant immunity modulation applicable in engineering disease-resistant crops. Plant Sci. 270:72-84.

Veronese, P., Nakagami, H., Bluhm, B., Abuqamar, S., Chen, X., Salmeron, J., Dietrich, R. A., Hirt, H., and Mengiste, T. 2006. The membrane-anchored BOTRYTIS-INDUCED KINASE1 plays distinct roles in Arabidopsis resistance to necrotrophic and biotrophic pathogens. Plant Cell 18:257-273.

Wingett, S. W., and Andrews, S. 2018. FastQ Screen: A tool for multigenome mapping and quality control. F1000 Res. 7:1338.

Zeng, R., Gao, S., Xu, L., Liu, X., and Dai, F. 2018. Prediction of pathogenesis-related secreted proteins from Stemphylium lycopersici. BMC Microbiol. 18:191.

Zhang, W., Corwin, J. A., Copeland, D. H., Feusier, J., Eshbaugh, R., Cook, D. E., Atwell, S., and Kliebenstein, D. J. 2019. Plant-necrotroph co-transcriptome networks illuminate a metabolic battlefield. eLife 8:e44279.

Zhang, Z. N., Wu, Q. Y., Zhang, G. Z., Zhu, Y. Y., Murphy, R. W., Liu Z., and Zou, C. G. 2015. Systematic analyses reveal uniqueness and origin of the CFEM domain in fungi. Sci. Rep. 5:13032.

Zhu, W., Ronen, M., Gur, Y., Minz-Dub, A., Masrati, G., Ben-Tal, N., Savidor, A., Sharon, I., Eizner, E., Valerius, O., Braus, G. H., Bowler, K., Bar-Peled, M., and Sharon, A. 2017a. BcXYG1, a secreted xyloglucanase from Botrytis cinerea, triggers both cell death and plant immune responses. Plant Physiol. 175:438-456.

Zhu, W., Wei, W., Wu, Y., Zhou, Y., Peng, F., Zhang, S., Chen, P., and Xu, X. 2017b. BcCFEM1, a CFEM domain-containing protein with putative GPI-anchored site, is involved in pathogenicity, conidial production, and stress tolerance in Botrytis cinerea. Front. Microbiol. 8:1807. 